\title{
SCN1A mutation spectrum in a cohort of Bulgarian patients with GEFS+ phenotype
}

\author{
Valentina Peycheva ${ }^{1 \odot}$, Nevyana Ivanova ${ }^{1 \odot}$, Kunka Kamenarova $^{1 \odot}$, \\ Margarita Panova ${ }^{2 \odot}$, Iliana Pacheva ${ }^{2 \odot}$, Ivan Ivanov ${ }^{2 \odot}$, Maria Bojidarova $^{3 \odot}$, \\ Genoveva Tacheva $^{3 \odot}$, Dimitar Stamatov ${ }^{3 \odot}$, Ivan Litvinenko ${ }^{3 \odot}$, Dimitrina Hristova ${ }^{4 \odot}$, \\ Daniela Deneva $^{5 \odot}$, Elena Rodopska ${ }^{5 \oplus}$, Elena Slavkova ${ }^{5 \oplus}$, Iliyana Aleksandrova ${ }^{5 \oplus}$, \\ Emil Simeonov ${ }^{6 \oplus}$, Petia Dimova ${ }^{7 \oplus}$, Veneta Bojinova ${ }^{5 \odot}$, Vanyo Mitev $^{1 \odot}$, \\ Albena Jordanova ${ }^{1,8 \odot}$, Radka Kaneva ${ }^{1 \oplus}$ \\ ${ }^{1}$ Department of Medical Chemistry and Biochemistry, Molecular Medicine Center, Medical University-Sofia, Sofia, Bulgaria, ${ }^{2}$ Department \\ of Pediatrics and Medical Genetics, Medical Faculty, Medical University-Plovdiv, Plovdiv, Bulgaria, ${ }^{3}$ Department of Pediatric Neurology, \\ University Pediatrics Hospital, Medical University- Sofia, Sofia, Bulgaria; Children Neurology Unit, ${ }^{4}$ Pediatrics Clinic, Acibadem City \\ Clinic Tokuda Hospital, Sofia, Bulgaria, ${ }^{5}$ Department of Neurology, University Hospital of Neurology and Psychiatry "St' Naum", \\ Clinic of Child Neurology, Medical University-Sofia, Sofia, Bulgaria, 'University Hospital "Alexandrovska", Pediatrics Clinic, Medical \\ University-Sofia, Sofia, Bulgaria, 'Epilepsy Surgery Center, University Hospital "St. Ivan Rilski", Medical University-Sofia, Sofia, \\ Bulgaria, ${ }^{8}$ VIB Department of Molecular Genetics, Molecular Neurogenomics Group, University of Antwerp, Antwerp, Belgium
}

\begin{abstract}
Background. Dravet syndrome (DS) is the most severe form of Generalized Epilepsy with Febrile Seizures plus (GEFS+) syndrome with a clear genetic component in $85 \%$ of the cases. It is characterized by fever-provoked seizure onset around six months of age and subsequent developmental deterioration later in life.
\end{abstract}

Methods. In the current study, 60 patients with fever-provoked seizures and suspicion either of GEFS+ (50 patients) or of DS (10 patients) were referred for SCN1A gene sequence analysis.

Results. SCN1A gene sequencing revealed clinically significant variants in 11 patients (18.3\%); seven pathogenic $(11.7 \%)$ and four likely pathogenic $(6.7 \%)$. Five of these variants have not been reported previously. Among the preselected group of ten DS patients, five had pathogenic SCN1A variants which confirmed diagnosis of DS. In four patients with preliminary diagnosis GEFS+, the detected SCN1A variant enabled us to specify the diagnosis of DS in these patients. Thus, SCN1A sequencing led to confirmation of the genetic diagnosis in 50\% (5/10) of DS patients, as well as clarification of the diagnosis of DS in $8 \%$ of GEFS+ patients (4/50).

In this study, four patients with truncating mutations had refractory seizures and additional psychomotor abnormalities. Additionally, pathogenic missense mutations were detected in three children with comparable phenotypes, which support the observations that missense mutations in critical channel function regions can cause a devastating epileptic condition.

Conclusions. This is the first systematic screening of SCN1A gene in our country, which expands the spectrum of $S C N 1 A$ variants with five novel variants from Bulgaria and demonstrates the clinical utility of confirmatory SCN1A testing, which helps clinicians make early and precise diagnoses. It is important for a better followup, choice of proper treatment, avoidance of development of refractory seizures and neuropsychological complications. Identification of pathogenic variants in SCN1A in the milder GEFS+ and severe DS cases, will help to offer adequate prenatal diagnosis and improve the genetic counselling provided to affected families.

Key words: Dravet syndrome, SCN1A mutation, GEFS+, seizures.

$凶$ Valentina Peycheva

valpey@mmcbg.org

Received 19th September 2019, revised 11st December 2019, accepted 15th January 2020. 
Dravet syndrome (DS) or Severe Myoclonic Epilepsy of Infancy (SMEI) is a well characterized epileptic encephalopathy in infants and has a clear genetic component in $85 \%$ of cases worldwide. ${ }^{1}$ It is defined by seizure onset around six months of age, along with the appearance of recurrent febrile status epilepticus (SE). These are fever-provoked generalized or focal, clonic or tonic-clonic seizures. Other seizure types, including myoclonic, atonic and atypical absence generally appear around 1 and 4 years of age. ${ }^{2}$ The seizures are often pharmacoresistant and from their second year of life the affected children develop cognitive, motor and behaviour impairments. ${ }^{3}$ Once appeared, the neuropsychological deficit is irreversible, does not respond to antiepileptic drugs (AEDs) and thus the DS prognosis is poor. DS is the most severe form of Generalized Epilepsy with Febrile Seizures plus (GEFS+) syndrome (OMIM 604233), which is a variable autosomal dominant epileptic condition that also associates with febrile and afebrile seizures, where the outcome is usually benign and the patients are sensitive to anti-epileptic treatment.

In the majority of DS cases (70-80\%) SCN1A gene is affected. ${ }^{4,5}$ SCN1A gene (OMIM 182389) encodes alpha subunit of voltage-gated sodium channel in GABAergic inhibitory interneurons, responsible for generation and propagation of action potentials in the brain. ${ }^{6}$ The spectrum of SCN1A mutations includes single nucleotide variations (SNVs) in $70-80 \%$ of DS patients, while patients harbouring intragenic or whole SCN1A gene deletions are much less. ${ }^{4,7}$ All of the mutations detected in milder GEFS+ cases are missense. ${ }^{8}$ Almost all SCN1A mutations in DS patients are de novo (95\%), although some of them are inherited from an affected parent, often with a milder phenotype. ${ }^{9}$ Analysis of genotype-phenotype correlations show that truncating mutations, partial or whole gene deletions as well as missense mutations localized in pore-forming regions (S5-S6) and in voltage-gated S4 segment cause complete loss of function of the protein (haploinsufficiency) and as a result more severe phenotype ${ }^{10,11}$, compared to missense mutations in the linker regions of the protein which cause milder

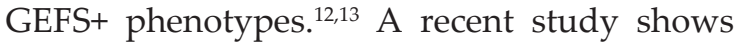
that truncating mutations cause earlier onset of seizures, appearance of other seizure types (myoclonic, atypical absence) and subsequent irreversible neuropsychological delay after seizure onset. ${ }^{4}$

In this sense, early and precise diagnosis of DS is critical, due to the high risk of SE and neuropsychological deficit, and that commonly prescribed antiepileptic sodium channel blockers may aggravate seizures in DS patients. ${ }^{14,15}$ Herein, we report the results from a systematic screening of SCN1A gene in a cohort of Bulgarian patients with epilepsy in the GEFS+ spectrum. Amongst the detected SCN1A mutations five have not been previously reported and we attempt here to make genotypephenotype correlations based on the predicted effect of the novel mutations on the encoded Nav1.1 protein structure and function.

\section{Material and Methods}

\section{Subjects}

In the current study a total of 60 patients were referred by clinicians from major neurology clinics in Bulgaria ( $\mathrm{n}=60 ; 28$ males, 32 females). Patients' clinical information, including type of seizures, the age at seizure onset, the frequency and duration of the convulsive seizures, as well as the response to administration of AEDs was gathered. The assessment of epileptic seizures was performed according to the revised diagnostic criteria of the International League Against Epilepsy classification. ${ }^{16}$

In the Dravet group, 10 patients were included, who had normal development before seizure onset, the occurrence of either fever-provoked or unprovoked partial, generalized seizures before the first year of life, appearance of other seizure types (myoclonic, atonic, absence), presence of intractable epilepsy, and a gradual psychomotor delay after the seizure onset, as well as presence of gait disturbances and other 
pyramidal signs. The remaining 50 patients who were not fully consistent with the accepted diagnostic criteria or had milder forms of GEFS+ were also tested for SCN1A mutations.

All participants and/or their legal guardians gave written informed consent. The study was conducted with the approval by Ethics Committee of Medical University of Sofia (Ethical approval number: 2011-2655).

\section{Genetic testing}

DNA was extracted from peripheral blood lymphocytes of patients and their parents (where available) using automatic Chemagic Magnetic Separation Module I system (Perkin Elmer, Germany). The quality and quantity of the DNA samples were determined using the NanoDrop ND-2000 spectrophotometer.

All 26 exons including exon/intron boundaries of SCN1A gene were amplified by Polymerase Chain Reaction (PCR) in 28 separated reactions using previously reported primer pairs. ${ }^{17}$ Sanger sequencing of the purified PCR products was conducted using ABI 3130xl (Applied Biosystems, Foster City, CA, USA). The sequences were analysed using $\mathrm{ABI}$ Sequencing Analysis v.5.3 and compared with reference sequence NG_011906.1. In the positive cases a segregation analysis was performed where parents' DNA was available. A total of 28 relatives were analysed (Table I).

\section{Variant analysis}

The candidate variants were examined using reference sequence NM_001165964 and public databases, including 1000G (http:// www.1000genomes.org), ExAc (http://exac. broadinstitute.org/), ClinVar (https://www. ncbi.nlm.nih.gov/clinvar), Human Gene Mutation Database - HGMD (www.hgmd. cf.ac.uk), as well as the database of Molecular genetics laboratory of the University of Antwerp (http://www.molgen.vib-ua.be/ SCN1AMutations/). Missense variants were analysed by three bioinformatic tools PROVEAN/SIFT, Polyphen2 and Mutation
Taster. The pathogenicity of splice-site variants was assessed by NetGene2 and Human Splicing Finder software in order to evaluate their potential effects. ${ }^{18-21}$ Variant interpretation and classification was carried out according to the criteria provided by American College of Medical Genetics and Genomics (ACMG) standards and guidelines. ${ }^{22}$

\section{Results}

Out of 60 patients, 22 carried sequence variations in SCN1A gene $(36.6 \%)$ (Table I). Among them a total of 18 variants were found, five of which were novel. Using ACMG criteria for variant classification, variants with clinical significance were detected in 11 patients $(18.3 \%)$ - seven pathogenic $(11.7 \%)$ and four likely pathogenic variants $(6.7 \%)$ (Table I). Five variants classified as likely benign, weak benign or benign were detected, among which three were recurrent (Table I). One benign variant, c.603-91G>A, represented a common polymorphism, associated with particular response to antiepileptic drug therapy. Last two of the detected variants were classified as variants of unknown significance (VUS) (c.4252$4 \mathrm{~A}>\mathrm{G}$ and p.Arg542Gln).

Among the preselected 10 patients with DS phenotype, five had SCN1A mutations (50\%) (Patients2, 6, 7, 8, 10). Four additional patients referred with presumptive diagnosis of GEFS+ were found to harbour pathogenic mutations in SCN1A (Patients 1,4, 5 and 9; see Tables I, II). Their epileptic condition was afterwards clearly specified as DS. Thus, SCN1A sequencing led to the genetic clarification of the diagnosis of DS in $8 \%(4 / 50)$. In two milder GEFS+ cases variants with clinical significance were found in 2 patients (patients 3 and 11) - one novel splicesite (c.2383-1G>A) and one missense mutation (p.Met1654Ile).

\section{Clinical summary of the patients with SCN1A mutations}

In all 22 patients with detected mutations, the main clinical feature was fever-provoked or 


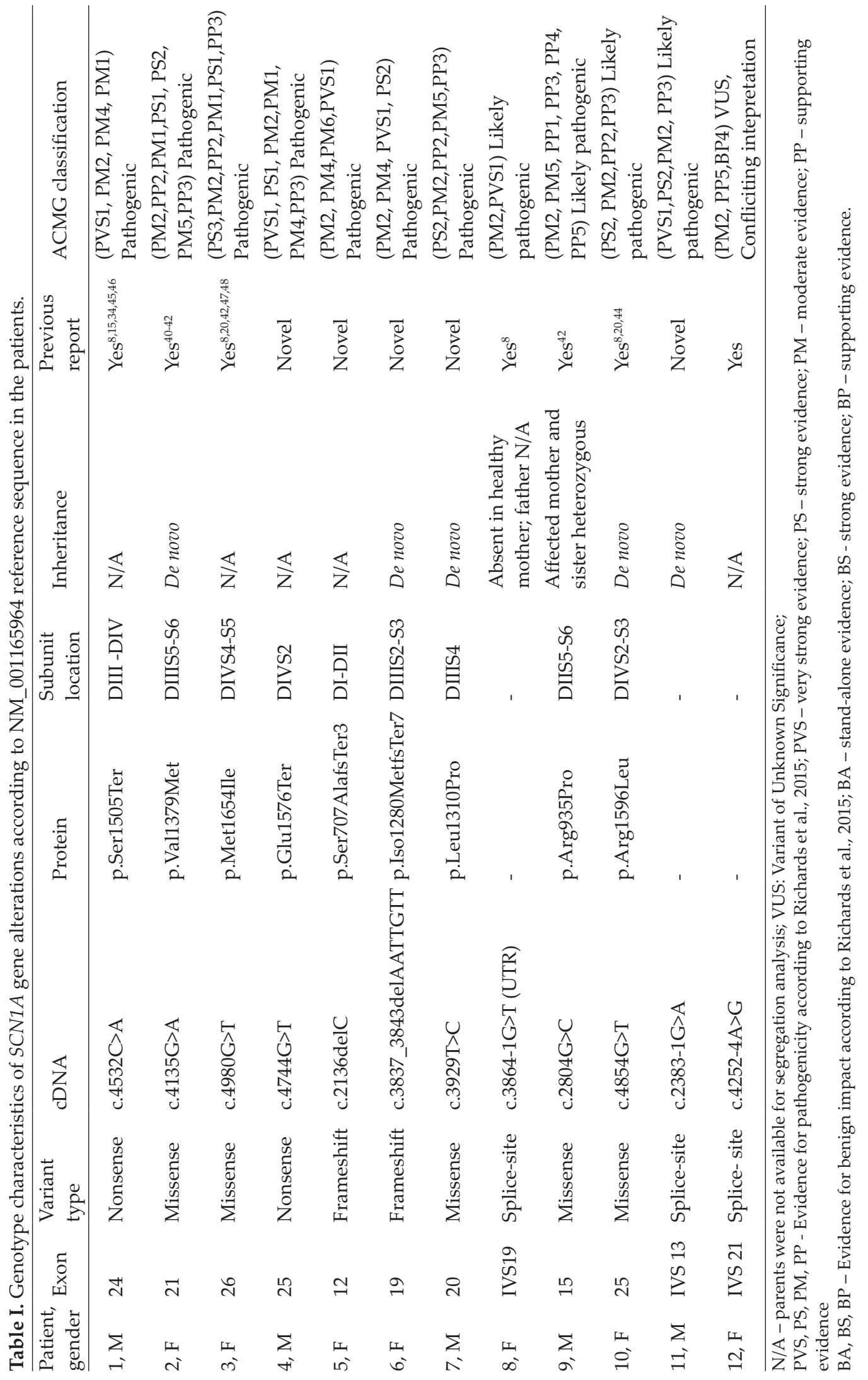




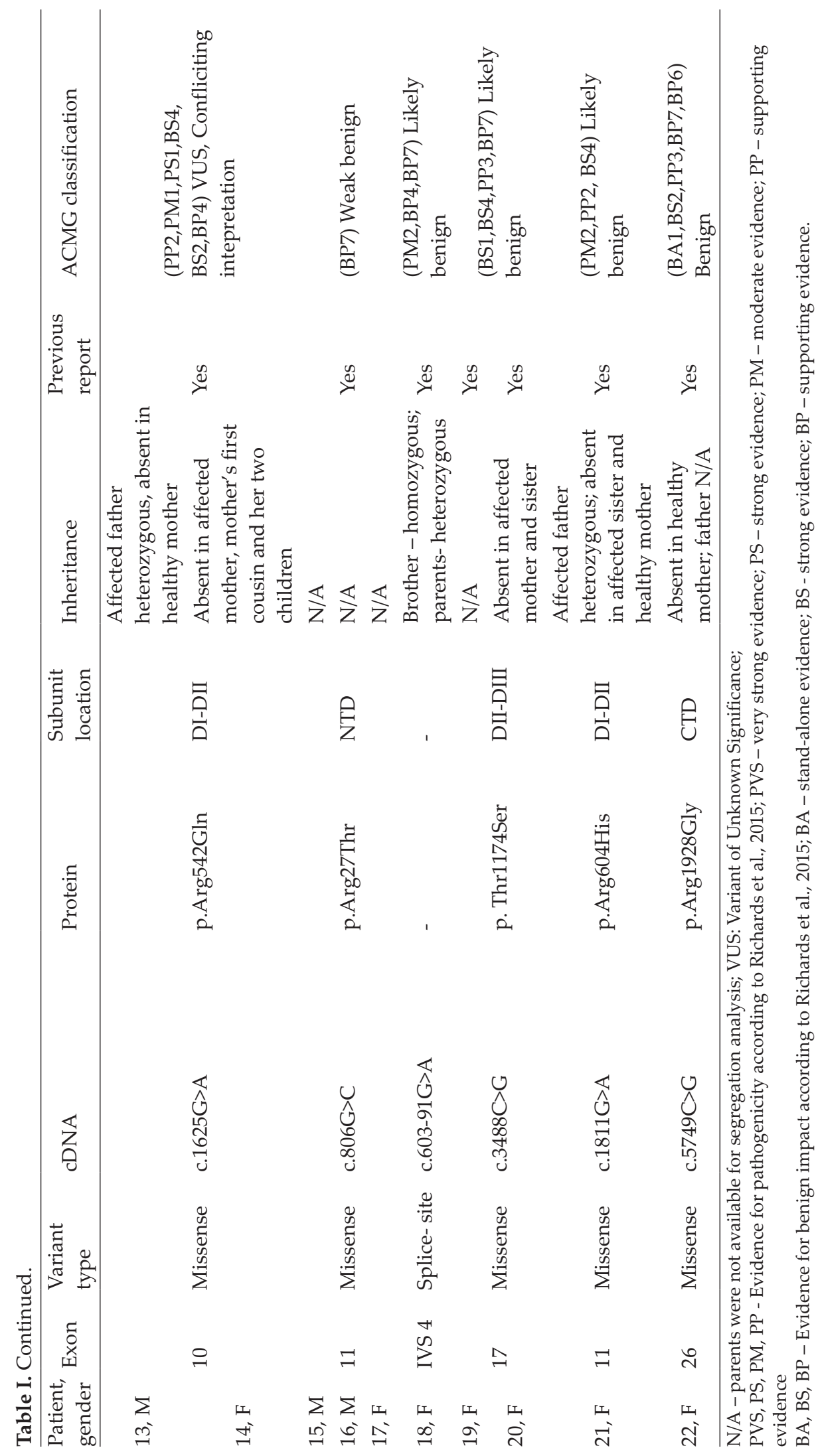


unprovoked generalized tonic-clonic seizures (GTCS) (Table II). In three patients additional myoclonic (patients 8 and 9), atonic and absences seizures (patient 7) are observed. In eight of the patients GTCS were preceded by focal unilateral seizures and subsequent second generalization. The seizure onset was between 3 months and 1 year and 8 months of age. Ten patients had positive family history and their relatives reported single febrile seizures (FS) in their childhood triggered by infectious disease and were remitted after 5 years of age. Eight patients exhibited at least one SE after the seizure onset; three patients referred as GEFS+ (Patients 1, 3, 9 ) and 5 patients as DS (Patients $2,6,7,8,10)$. In eight patients subsequent developmental and cognitive delay were observed - four DS patients $(2,6,7$ and 8$)$ and four patients referred as GEFS+ $(1,4,5$, and 9). Additional dysmorphic features were observed in patients 7 and 8 (Table II).

\section{Summary of the genotype information}

Out of 11 clinically significant SCN1A mutations five were missense, two affected conservative splice sites and four were truncating mutations ( 2 nonsense and 2 frameshift deletions) (Table I). None of them were present in public variant databases, like ExAc or 1000 Genomes. Five of the identified pathogenic variants occurred de novo ( 3 missense, 1 splice site and 1 frameshift deletion) and one missense variant was inherited from the mother. In five patients segregation analysis was not performed because parents were not available (Table I). Five mutations have not been reported before - one nonsense (p.Glu1576Ter), two frameshift deletions (p.Ser707AlafsTer3, p.Iso1280MetfsTer7), one missense (p.Leu1310Pro) and one splice-site mutation (c.2383-1G>A) (Table I).

\section{Genotype/phenotype correlations}

Among the patients with detected clinically significant SCN1A variation, four (Patients 1, $4,5,6)$ were truncating mutations leading to premature stop of the synthesis of intact protein and are found in patients with severe epileptic phenotype (Fig. 1, Table II). Three of them were reported for the first time in the current study; p.Glu1576Ter, p.Ser707AlafsTer3 and p.Iso1280MetfsTer7 (TableI). All of the truncating mutations were found in children with earlier seizure onset (before age of one year), refractory epilepsy, no effect or partial response to the AED treatment and start of neuropsychological delay after seizure onset (Table II). Patients 1 and 5

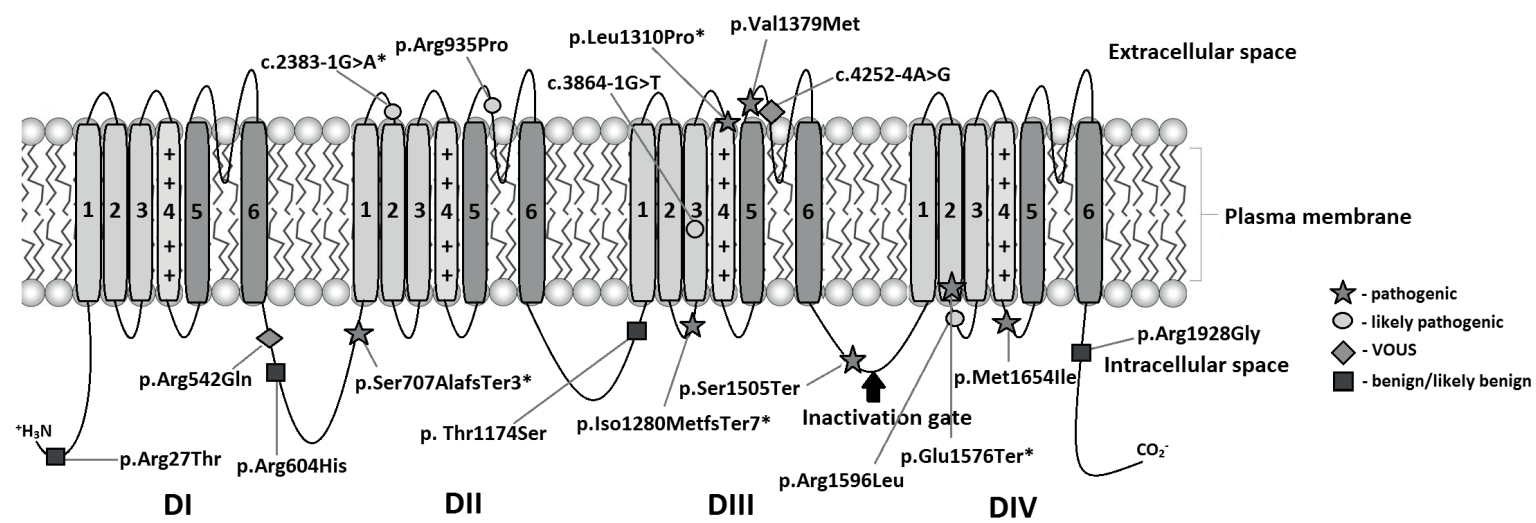

Fig. 1. Schematic representation Nav1.1 protein structure, showing four homologous domains (DI-DIV), each with six transmembrane segments (S1-S6). Segments five and six (S5, S6) form the ion channel pore and segment four is the voltage sensor. Segments S1-S3 have structural role for the protein. Segment five and six of each homologous domain and the re-entrant loops between them form the pore. The intracellular loop between DIII and DIV (indicated by an arrow) forms an inactivation gate, which is necessary for fast inactivation of the channel. On the diagram are illustrated the location and distribution of identified mutations (except the intronic variant c.603-91G>A) represented with different shapes. 


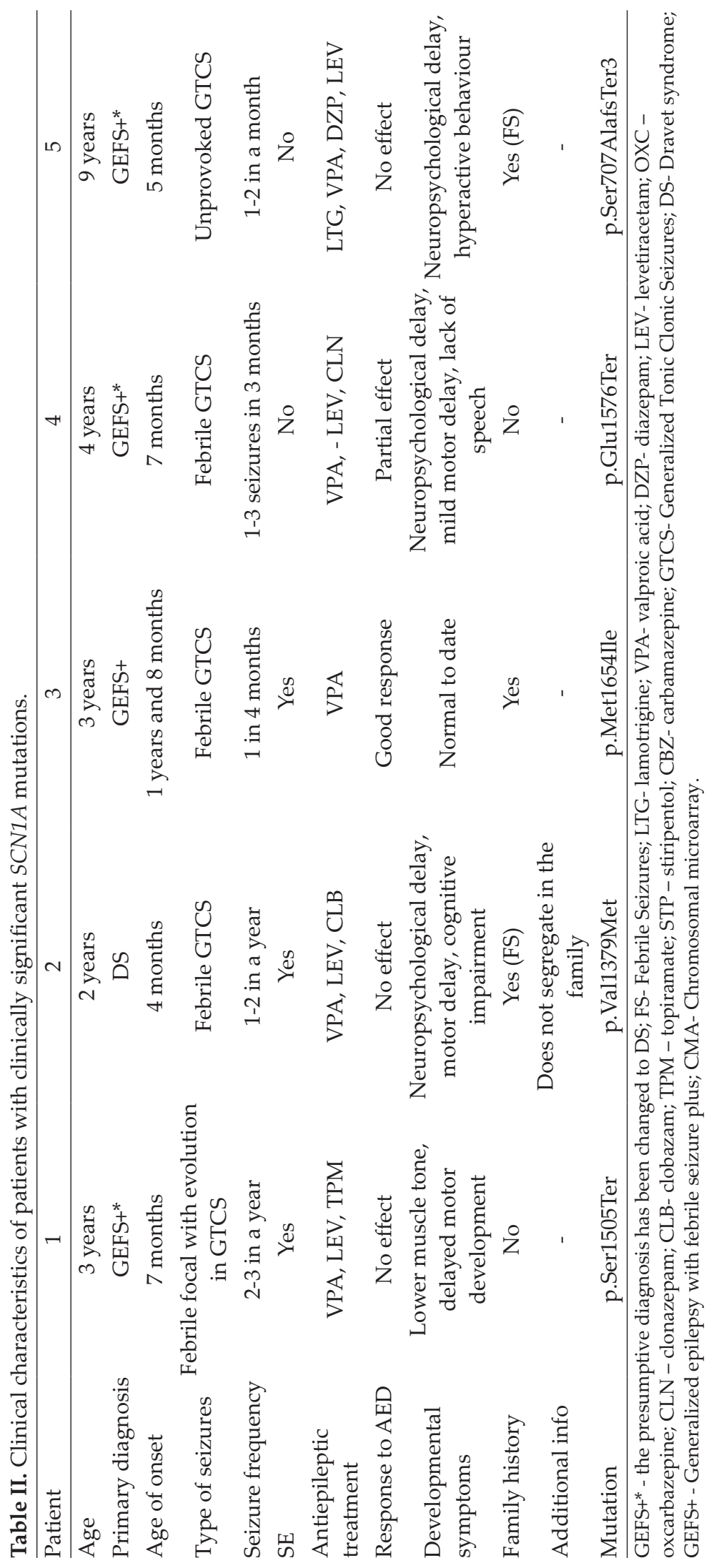




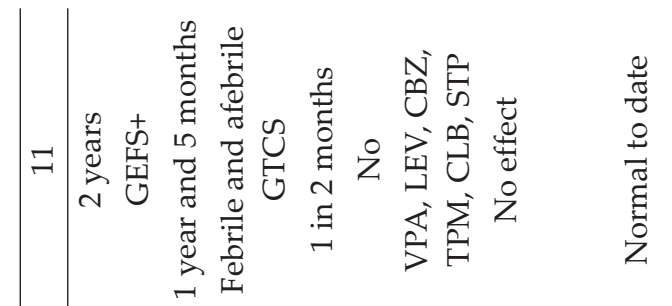

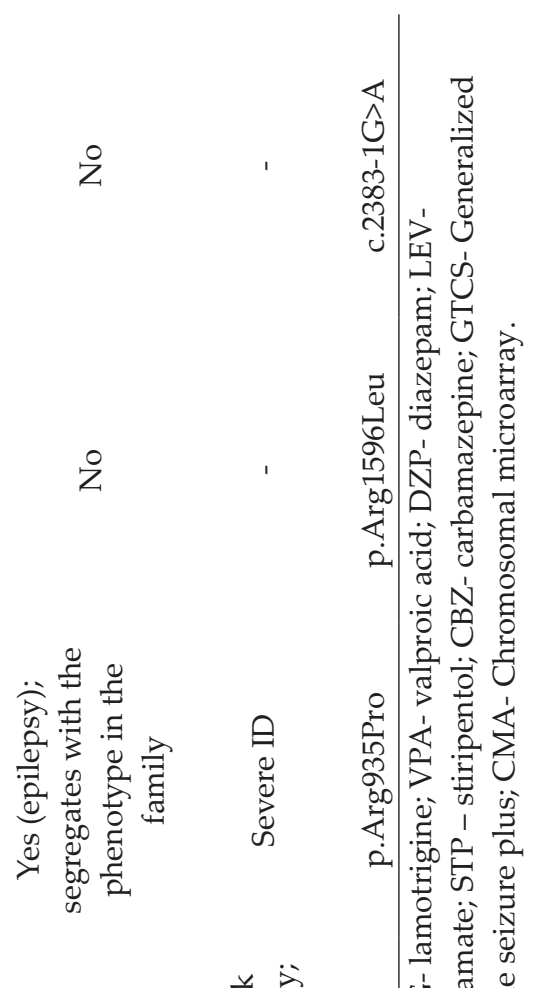

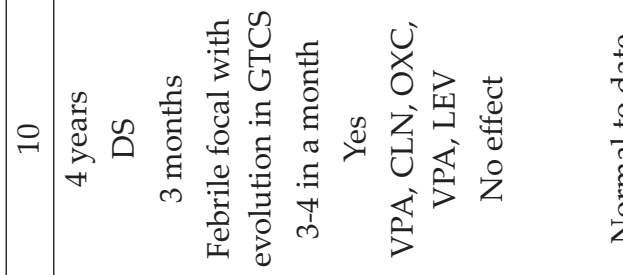

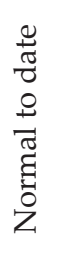

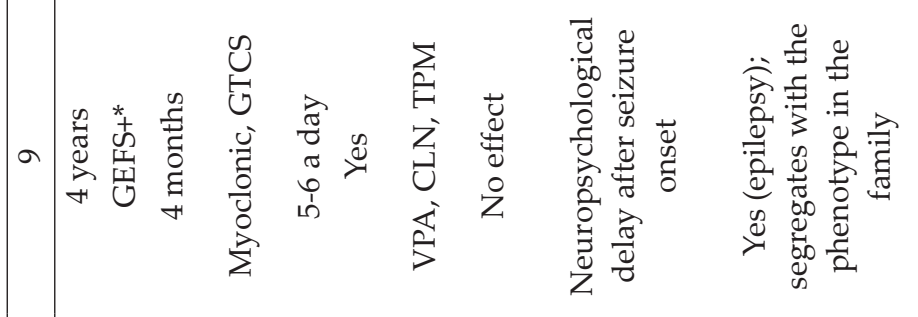

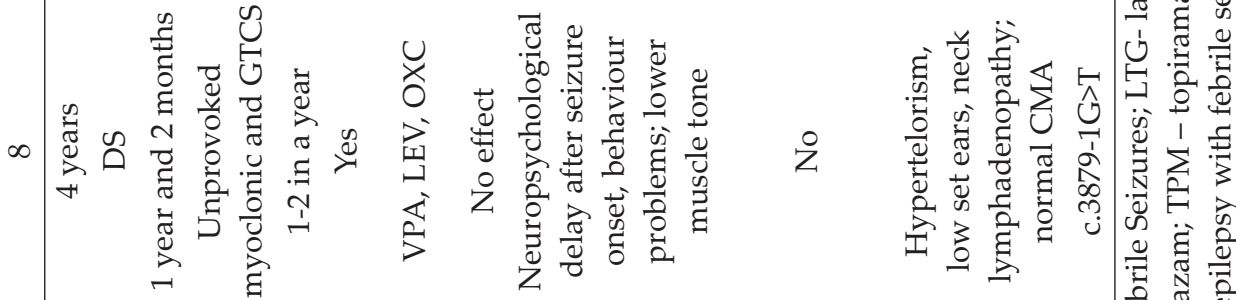

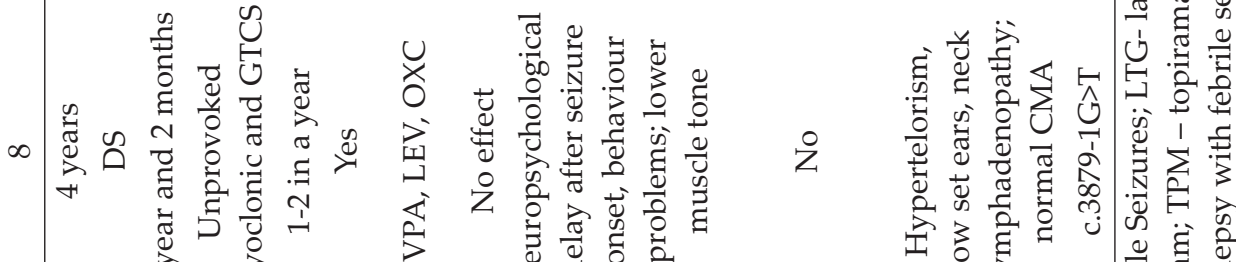

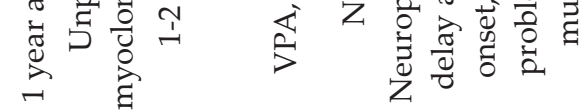

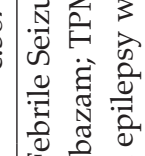

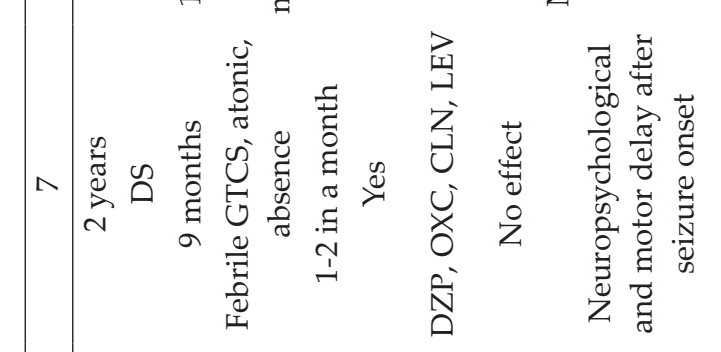

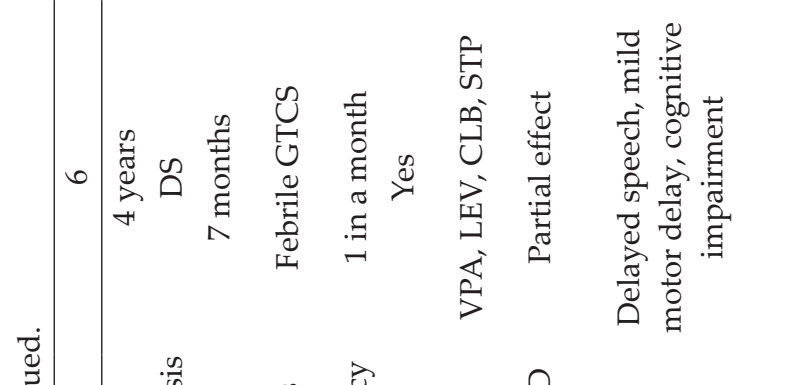
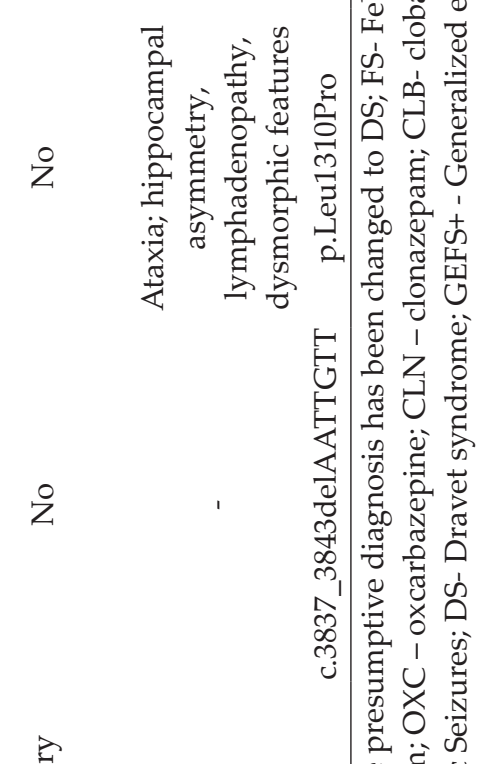

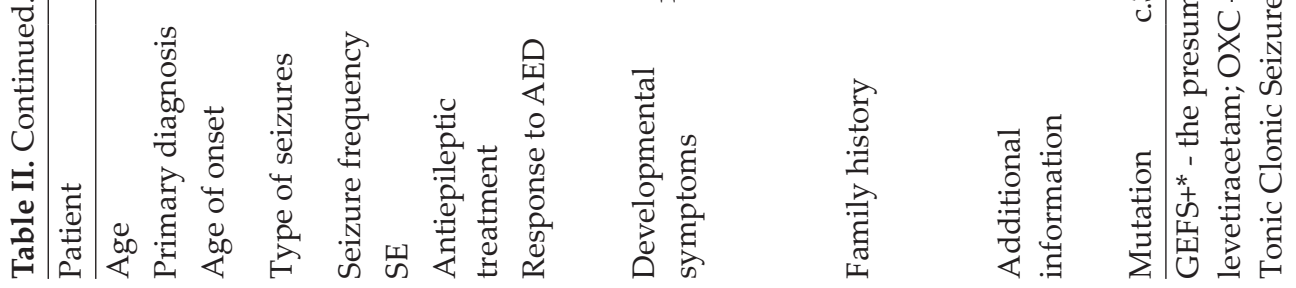


who carried nonsense p.Ser1505Ter mutations and frameshift p.Ser707AlafsTer3 respectively, did not respond to the administration of several AED (Table II). In the other two patients (4 and 6) with truncating mutations (Table I) a partial effect of AED treatment was observed - the seizures became less frequent with appearance of 1 or 2 convulsions annually. However, their neurological impairment persisted - both of them did not speak and exhibited cognitive and motor delay.

Two splice-site mutations detected in the current study are assessed by prediction tools as likely pathogenic mutations - c.3864$1 G>T$ and c.2383-1G>A (Table I). The novel splice site variant c.2383-1G>A was detected in a child with pharmaco-resistant GTCS and occurred de novo. It is located in the canonical splice-acceptor site of intron 13 and according to NetGene2 and Human Splicing finder prediction software, there is high confidence for the use of the first alternative AG dinucleotide located 23 nucleotides downstream in exon 14 . The SCN1A splice-site variant c.3864-1G>T has similar prediction parameters. It is located in the canonical splice-acceptor site of intron 19 (Table I) and was also detected in a 4-year-old child with refractory epilepsy, later seizure onset and presence of neuropsychological delay after seizure onset, as well as behaviour problems (Table II). According to prediction tools, there is high possibility for usage of alternative AG dinucleotide located downstream in exon 20.

Among the missense mutations, four were previously reported in other patients with comparable phenotype (Table I) and one is novel (p.Leu1310Pro in Patient 7). The p.Arg935Pro and p.Val1379Met mutations are located in highly conserved S5-S6 linker region, which is part of the pore forming region. The p.Leu1310Pro is located on voltage sensor segment of the third domain. All three missense mutations represent non-conservative AA substitutions with a potentially significant impact on physicochemical properties of the channel and are located in important domains for the channel function (Fig. 1). As a result, an early seizure onset and presence of additional to GTCS types of seizures (myoclonic, atonic, absence) were observed in patients 2, 7 and 9 (Table II). Moreover, these patients show refractory epilepsy to multiple AED applied and a neuropsychological delay after seizure onset. Patient 7 additionally exhibited ataxic gait and dysmorphic features (Table II). The p.Arg1596Leu(in Patient 10) and p.Met1654Ile (in Patient3) mutations are located in the cytoplasmfacing loop, connecting S2-S3 segments and S4S5 segments of the fourth domain, respectively (Fig. 1). Their localization outside of the poreforming region probably results in a milder effect to the channel's function. In patient 10, who carried de novo p.Arg1596Leu a normal psychomotor development was observed on the day of examination. Patient 3 with detected p.Met1654Ile exhibited later seizure onset, as well as good response to valproic acid (VPA) and normal psychomotor development (Table II). For all clinically significant missense mutations the applied prediction tools showed that they had a demolishing effect to the function of the subunit.

All benign missense variants are situated in either linker regions or $\mathrm{N}$ - or C-terminal domains (NTD, CTD) (blue colored circles Fig. 1). In these patients a later seizure onset is observed (median 2 years of age) and the mutations do not segregate with the phenotype in the family.

\section{Discussion}

This is the first large-scale screening of SCN1A in fever-provoked epilepsy in our country. To date, different type of SCN1A mutations in single cases have been reported..$^{23-25}$

In the current study, a total of 7 pathogenic SNVs among 60 patients were detected (11.7\%). Likely pathogenic mutations were found in four patients $(6.7 \%)$, which makes an overall diagnostic yield of $18.3 \%$. The observed diagnostic yield of pathogenic changes $(11.7 \%)$ is in line with those recently reported cohorts of 
patients with GEFS+ syndrome and pathogenic SCN1A mutations $(10 \%)^{4,5}$ As a result of our study, a pathogenic $S C N 1 A$ variant was detected in five patients with presumptive diagnosis of DS. In four additional patients $(1,4,5,9)$ with preliminary diagnosis of GEFS+ mutational analysis revealed two nonsense mutations; p.Ser1505Ter, p.Glu1576Ter, of which the latter is novel (Table I), one novel frameshift deletion p.Ser707AlafsTer3, as well as one missense mutation p.Arg935Pro affecting the poreforming linker S5-S6 segment from domain DIII (Table I). Thus, we were able to specify the genetic diagnosis in $50 \%$ of DS patients, as well as to clarify the diagnosis of DS in $8 \%$ of GEFS+ patients. The observed diagnostic rate is lower than those reported in the literature in similar cohorts of DS patients $(70-80 \%) .{ }^{4}$ This partly might be due to the small sample size of DS patients $(n=10)$, as well as comparatively loose selection criteria applied. Furthermore, SCN1A negative DS patients may have resulted from mutations in other epilepsy-associated genes, such as SCN1B, HCN1, STXBP1, CHD2, $G A B R A 1$, and $G A B R G 2$, that were not analyzed in the current study. ${ }^{26-28}$ It is important to perform large-scale genomic approaches like clinical or whole exome sequencing in order to confirm this hypothesis.

The classification and interpretation of pathogenicity of the detected variants was carried out in accordance with the criteria provided by ACMG. ${ }^{22}$ The authors provided two sets of criteria: one for classification of pathogenic and likely pathogenic variants and one for classification of benign and likely benign variants. Each of the criteria was divided in different categories - very strong (PVS1), strong (PS1-4), moderate (PM1-6) and supporting (PP1-5) for pathogenic criteria and stand-alone (BA1), strong (BS1-4) or supporting (BP1-6) for benign criteria. ${ }^{22}$ The numbering within each category does not give any precedence of some criteria over others, but rather is used for differentiation. Given the available information for a particular variant, either taking into account previously published data, or data obtained from the current case under investigation, the combination of these criteria gives the final variant interpretation and classification. All criteria for variant assessment and the scoring rules for their combination are explained in detail by Richards et al., 2015. ${ }^{22}$ The highly possible consequence of the novel c.2383$1 \mathrm{G}>$ A splice-site mutation detected in patient 11 might be a deletion of 23 nucleotides from exon 14 and formation of stop codon after four amino acids (AA) in downstream direction of the second domain from the Nav1.1 protein (Fig. 1) (c.2383_2406del; p.Val795LysfsTer4). The effect of the other splice site mutation c.3879-1G>T was also predicted to result in deletion of 68 nucleotides from exon 20 and frameshift deletion of 15 AA in S3 from the third domain (DIII) the Nav1.1 protein (p.Val1283AspfsTer15). Another possible consequence is the usage of the spliceacceptor site of some of the adjacent introns, subsequent exon skipping and activation of nonsense-mediated mRNA decay process with resultant non-productive translation. Both patient carriers 8 and 11 show severe refractory epilepsy and neuropsychological deterioration at age of examination (Table II). However, to clarify the potential pathogenicity of the splice SCN1A variants it is important to investigate their effect at the RNA level, as well as to perform a follow up of patients' development at a later stage.

In the current study all patients with truncating mutations $(1,4,5,6)$ had seizures refractory to AEDs and additional psychomotor abnormalities. Thus, we here confirm previously reported observations that truncating mutations (nonsense, frameshift, some splice site mutations) result in loss of function of the protein and are responsible for the occurrence of more severe epileptic phenotypes. ${ }^{11,29-30}$ It is known that individuals harbouring missense mutations, in which the aberrant copy of the gene is expressed despite its altered function, show milder phenotypes of the GEFS+ spectrum..$^{29,34}$ Some missense mutations exclusively cause severe consequences, because they are localized in critical channel function 
regions and/or the mutated AA has significantly different physicochemical properties, compared to the wild type AA. ${ }^{35}$ Pathogenic changes in amino acid polarity have been detected in voltage sensor (S4) and ion-pore regions from Nav1.1 protein and are associated with the DS phenotype rather than milder GEFS+ syndrome. ${ }^{11,13,35}$ In our study, the novel mutation p.Leu1310Pro is localized in $\alpha$ helix motif of voltage sensor S4 in domain DIII (Fig. 1). The wild type and mutant amino acids differ in structure and in this sense the substitution of leucine with proline leads to conformational change of the polypeptide chain, which might lead to disruption of the normal function of the voltage sensor segment. Patient 7 exhibited severe epileptic phenotype including refractory GTCS, ataxia and neuropsychological delay after seizure onset (Table II). The application of four antiepileptic drugs had no effect on the seizure frequency (Table II).

Additionally, one variant classified as likely pathogenic missense mutation (p.Arg935Pro) also changes the polarity of the AA residue in the pore forming S5-S6 DII segment of the Nav1.1 protein (Fig. 1). p.Arg935Pro segregates with the disease phenotype in the affected family - it is present in the sister with myoclonic seizures and moderate intellectual disability (ID), and in the mother, who has history of epilepsy in early childhood (Table I). The observed phenotypic heterogeneity is a characteristic phenomenon in dominant genetic epilepsies in general and might be due to individual epigenetic factors' influence or the presence of gene modifiers, which modulate the effect of the mutation via unrevealed pathomechanism. ${ }^{36-38}$ Patient 9 showed classical manifestation of DS - refractory seizures, which evolve in neuropsychological delay and later in life in ID (Table II). This might be due to impaired channel function as a result of the different chemical nature and the size of proline, compared to arginine. Moreover, proline disrupts the interaction between the protein and the lipid phase of the membrane, which additionally may contribute to the abnormal function of the channel. To date, p.Arg935Pro was reported in a single Chinese patient with DS. ${ }^{43}$ Mutations, defined as pathogenic and affecting the same AA, but different substitution (p.Arg935His, p.Arg935Cys), were reported in patients with DS and severe psychomotor delay, as well as in borderline DS cases. ${ }^{8,39-41}$

Patient 2, a 2-year-old boy also showed refractory seizures and severe psychomotor delay (Table II). He was found to have pathogenic missense mutation p.Val1379Met affecting the pore forming S5-S6 re-entrant loop of domain DIII with an important role for the proper function of the NaV1.1. subunit (Fig. 1). Similarly, in the other two cases with missense mutations (patients 7 and 9) the AA substitution was non-conservative. Leucine is hydrophobic AA, while methionine is sulfur-containing AA and has different spatial and physicochemical properties, compared to the wild type leucine. So far, this variant has been reported as pathogenic only in DS cases. ${ }^{42-44}$ In all of them, including in our case the mutation appeared de novo. At first, patient 2 showed a temporarily good response to the administration of VPA, but subsequently his development gradually declined. The addson therapy with levetiracetam and clobazam failed to cease the seizures and at the moment the child exhibits fever-provoked GTCS, gait disturbances and cognitive delay (Table II).

The three cases described (2, 7 and 9) support the observations that missense mutations critical for the channel function regions can cause loss of function of the protein and as a result - devastating epileptic phenotype..$^{12,35}$ Patient 10, a 4-year-old girl was a carrier of de novo missense SCN1A variant (p.Arg1596Leu) classified according ACMG criteria as likely pathogenic. At the time of examination patient 10 exhibited refractory epilepsy to multiple AED and normal development (Table II). The mutation was reported in single DS patient $t^{8}$, as

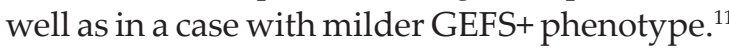
A different AA substitution at the same position was described in patient with cryptogenic focal epilepsy, moderate ID and ataxia ${ }^{45}$, as well as in a patient with classical GEFS+ features, normal 
development and good response to AEDs. ${ }^{46}$ Unfortunately, we cannot perform a follow-up of the patient's clinical outcome, because of lack of contact with the family.

In the current study, four patients did not have a family history of epilepsy and the segregation analysis revealed de novo SCN1A variant (patients 6, 7, 10, 11; see Tables I, II). In contrast, one de novo SCN1A pathogenic variant does not segregate with the epilepsy phenotype in the family (see Tables I, II, patient 2) - the parents experienced single FS in the past and did not carry the variant. This may be explained by the polygenic nature of FS. It is possible that the parents carry additional mutation in another epilepsy associated gene, which is the genetic cause or acts like a predisposition factor for the febrile seizures, observed in them. This factor might have also contributed in combination with the SCN1A mutation to their child's phenotype. Examples of specific genetic modifier genes, mutations in which modulate the effect of the corresponding $S C N 1 A$ variant are $S C N 8 A$, SCN9A, CACNB4..$^{47-49}$ A missense SCN9A variant was found in DS patient from Caucasian origin inherited from the asymptomatic mother with history of FS. This patient also carried de novo frameshift mutation in SCN1A and the authors concluded, that SCN9A has a contributing role to the DS phenotype. ${ }^{49}$ Similar findings were reported in cases with detected SCN1A mutation in combination with CACNB4 mutation, the latter inherited from asymptomatic father with FS in the past. The CACNB4 mutation resulted in increased neurotransmitter release in the excitatory neurons under insufficient inhibitory neurons caused by the nonfunctional Nav1.1 channel, caused by the SCN1A mutation. ${ }^{48}$ However, to prove this hypothesis in our case, it is necessary to apply a wide-range of technology, such as next-generation sequencing, in order to identify this additional FS-related genetic modifier in family 2 .

It is now known that in a family with a particular genetic disease the recurrence risk for a first degree relative to have the same disease caused by a de novo mutation is higher than that of the general population and is estimated at $1-4 \% .{ }^{50}$ Around $80 \%$ of the inherited de novo germline point mutations arise from the paternal allele and advanced paternal age at conception has been accepted as the major factor linked to the de novo mutations in the offspring, both at the population level and within the same family. ${ }^{51,52}$ In particular, $10 \%$ of DS patients have inherited pathogenic $S C N 1 A$ variant from an asymptomatic or mildly affected parent., Furthermore, $7 \%$ of DS patients have seemingly healthy parents with germline mosaicism which increases the risk for passing the SCN1A mutation to the offspring. ${ }^{53}$ This high incidence can increase the risk further and has an important value for the genetic counselling of the affected families. In order to prevent recurrence in such families it is recommended to perform prenatal diagnosis either by chorion villus sampling or amniocentesis, depending on the week of pregnancy.

The results of the current study are important in three aspects. Firstly, they broaden the SCN1A mutation spectrum with novel variants from Bulgaria. Secondly, they can guide the choice of medication, avoiding sodium channel blockers (lamotrigine, carbamazepine, phenytoin) which can provoke seizure aggravation in classical DS phenotypes. The danger of seizure aggravation is usually ignored in "milder" GEFS+ cases examined before age of 2 years with undetected loss-of-function mutations, because of the lack of knowledge for the evolution of the syndrome, based only on clinical examination. These cases may have the same genetic basis as the classical DS and may have similar AEDs response. In this sense, an early and precise genetic diagnosis is critical, because it will help the clinician to choose AEDs punctually and prevent the development of intractable epilepsy. Our results demonstrate the clinical utility of confirmatory SCN1A testing, which helps the clinician to make appropriate assessment about the diagnosis. And finally, identification of pathogenic SCN1A variants in severe DS, as well as the milder GEFS+ phenotypes, will 
help to offer an adequate prenatal diagnosis and improve the genetic counselling offered to affected families.

\section{Acknowledgements}

We want to thank all the families participating in the study. Acknowledgements to Felitsiya Shakola, PhD for performing bioinformatics analysis of the SCN1A variants. This work was supported by the National Science Fund, Ministry of Education and Science, Bulgaria [grant number DTK67/2009], [grant number DUNK 01/2/2009].

\section{REFERENCES}

1. Guerrini R. Dravet syndrome: the main issues. Eur J Paediatr Neurol 2012; 16(Suppl 1): S1-S4.

2. Dravet C, Bureau M, Oguni H, Fukuyama Y, Çokar O.Severe myoclonic epilepsy in infancy (Dravet syndrome). In: Roger J, Bureau M, Dravet C, Genton P, Tassinari CA, Wolf P (eds). Epileptic Syndromes in Infancy, Childhood and Adolescence (4th ed). Montrouge: John Libbey Eurotext, 2005; 4: 89-113.

3. Ragona F, Granata T, Dalla Bernardina B, et al. Cognitive development in Dravet syndrome: a retrospective, multicenter study of 26 patients. Epilepsia 2011; 52: 386-392.

4. Marini C, Scheffer I, Nabbout R, et al. The genetics of Dravet syndrome. Epilepsia 2011; 52(Suppl 2): 24-29.

5. McTague A, Howell KB, Cross JH, Kurian MA, Scheffer IE. The genetic landscape of the epileptic encephalopathies of infancy and childhood. Lancet Neurol 2016; 15: 304-316.

6. Trimmer JS, Rhodes KJ. Localization of voltagegated ion channels in mammalian brain. Annu Rev Physiol 2004; 66: 477-519.

7. Suls A, Claeys KG, Goossens D, et al. Microdeletions involving the SCN1A gene may be common in SCN1A-mutation-negative SMEI patients. Hum Mutat 2006; 27: 914-920.

8. Depienne C, Trouillard O, Saint-Martin C, et al. Spectrum of SCN1A gene mutations associated with Dravet syndrome: analysis of 333 patients. J Med Genet 2009; 46: 183-191.

9. Kimura K, Sugawara T, Mazaki-Miyazaki E, et al. A missense mutation in SCN1A in brothers with severe myoclonic epilepsy in infancy (SMEI) inherited from a father with febrile seizures. Brain Dev 2005; 27: 424-430.
10. Nicita F, Spalice A, Papetti L, et al. Genotypephenotype correlations in a group of 15 SCN1Amutated Italian patients with GEFS+ spectrum (seizures plus, classical and borderline severe myoclonic epilepsy of infancy). J Child Neurol 2010; 25: 1369-1376.

11. Zuberi SM, Brunklaus A, Birch R, Reavey E, Duncan J, Forbes GH. Genotype-phenotype associations in SCN1A-related epilepsies. Neurology 2011; 76: 594600.

12. Kanai K, Hirose S, Oguni $H$, et al. Effect of localization of missense mutations in SCN1A on epilepsy phenotype severity. Neurology 2004; 63: 329-334.

13. Kanai K, Yoshida S, Hirose S, et al. Physicochemical property changes of amino acid residues accompany missense mutations in SCN1A affect the epilepsy phenotype severity. J Med Genet 2009; 46: 671-679.

14. Löscher W. Preclinical assessment of proconvulsant drug activity and its relevance for predicting adverse events in humans. Eur J Pharmacol 2009; 610: 1-11.

15. Takayama R, Fujiwara T, Shigematsu H, et al. Longterm course of Dravet syndrome: a study from an epilepsy center in Japan. Epilepsia 2014; 55: 528-538.

16. Berg A, Berkovic S, Brodie MJ, et al. Revised terminology and concepts for organization of seizures and epilepsies: report of the ILAE Commission on Classification and Terminology, 2005-2009. Epilepsia 2010; 51: 676-685.

17. Claes L, Del-Favero J, Ceulemans B, Lagae L, Van Broeckhoven C, De Jonghe P. De novo mutations in the sodium-channel gene SCN1A cause severe myoclonic epilepsy of infancy. Am J Hum Genet 2001; 68: 1327-1332.

18. Desmet O, Hamroun D, Lalande M, Collod-Béroud G, Claustres M, Béroud C. Human Splicing Finder: an online bioinformatics tool to predict splicing signals. Nucleic Acids Res 2009; 37: e67.

19. Adzhubei A, Schmidt S, Peshkin L, et al. A method and server for predicting damaging missense mutations. Nat Methods 2010; 7: 248-249.

20. Schwarz JM, Cooper DN, Schuelke M, Seelow D. MutationTaster2: mutation prediction for the deepsequencing age. Nat Methods 2014; 11: 361-362.

21. Choi Y, Chan AP. PROVEAN web server: a tool to predict the functional effect of amino acid substitutions and indels. Bioinformatics 2015; 31: 2745-2747.

22. Richards S, Aziz N, Bale S, et al; ACMG Laboratory Quality Assurance Committee. Standards and guidelines for the interpretation of sequence variants: a joint consensus recommendation of the American College of Medical Genetics and Genomics and the Association for Molecular Pathology. Genet Med 2015; 17: 405-423. 
23. Yordanova I, Todorov $\mathrm{T}$, Dimova $\mathrm{P}$, et al. One novel Dravet syndrome causing mutation and one recurrent MAE causing mutation in SCN1A gene. Neurosci Lett 2011; 494: 180-183.

24. Suls A, Velizarova R, Yordanova I, et al. Four generations of epilepsy caused by an inherited microdeletion of the SCN1A gene. Neurology 2010; 75: 72-76.

25. Dimova P, Yordanova I, Bojinova V, Jordanova A, Kremenski I. Generalized epilepsy with febrile seizures plus: novel SCN1A mutation. Pediatr Neurol 2010; 42: 137-140.

26. Patino A, Claes R, Lopez-Santiago LF, et al. A functional null mutation of SCN1B in a patient with Dravet syndrome. J Neurosci 2009; 29: 10764-10778.

27. Suls A, Jaehn A, Kecskés A, et al ; EuroEPINOMICS RES Consortium. De novo loss-of-function mutations in CHD2 cause a fever-sensitive myoclonic epileptic encephalopathy sharing features with Dravet syndrome. Am J Hum Genet 2013; 93: 967-975.

28. Carvill L, Weckhuysen S, McMahon JM, et al. GABRA1 and STXBP1: novel genetic causes of Dravet syndrome. Neurology 2014; 82: 1245-1253.

29. Claes LR, Deprez L, Suls A, et al. The SCN1A variant database: a novel research and diagnostic tool. Hum Mutat 2009; 30: E904-E920.

30. Meng H, Xu HQ, Yu L, et al. The SCN1A mutation database: updating information and analysis of the relationships among genotype, functional alteration, and phenotype. Hum Mutat 2015; 36: 573-580.

31. Brigo F, Storti M. Antiepileptic drugs for the treatment of severe myoclonic epilepsy in infancy. Cochrane Database Syst Rev 2013; 11: CD010483.

32. Cho MJ, Kwon SS, Ko A, et al. Efficacy of stiripentol in Dravet syndrome with or without SCN1A mutations. J Clin Neurol 2018; 14: 22-28.

33. Ishii A, Watkins JC, Chen D, Hirose S, Hammer MF. Clinical implications of SCN1A missense and truncation variants in a large Japanese cohort with Dravet syndrome. Epilepsia 2017; 58: 282-290.

34. Sugawara T, Mazaki-Miyazaki E, Fukushima K, et al. Frequent mutations of SCN1A in severe myoclonic epilepsy in infancy. Neurology 2002; 58: 1122-1124.

35. Chen YJ, Shi YW, Xu HQ, et al. Electrophysiological differences between the same pore region mutation in SCN1A and SCN3A. Mol Neurobiol 2015; 51: 1263-1270.

36. Depienne C, Trouillard O, Gourfinkel-An I, et al. Mechanisms for variable expressivity of inherited SCN1A mutations causing Dravet syndrome. J Med Genet 2010; 47: 404-410.
37. Guerrini R, Cellini E, Mei D, et al. Variable epilepsy phenotypes associated with a familial intragenic deletion of the SCN1A gene. Epilepsia 2010; 51: 2474-2477.

38. Goldberg-Stern H, Aharoni S, Afawi Z, et al. Broad phenotypic heterogeneity due to a novel SCN1A mutation in a family with genetic epilepsy with febrile seizures plus. J Child Neurol 2014; 29: 221226.

39. Xu X, Zhang Y, Sun H, et al. Early clinical features and diagnosis of Dravet syndrome in 138 Chinese patients with SCN1A mutations. Brain Dev 2014; 36: 676-681.

40. Berkovic S, Harkin L, McMahon JM, et al. De-novo mutations of the sodium channel gene SCN1A in alleged vaccine encephalopathy: a retrospective study. Lancet Neurol 2006; 5: 488-492.

41. Fukuma G, Oguni H, Shirasaka Y, et al. Mutations of neuronal voltage-gated $\mathrm{Na}+$ channel $\alpha 1$ subunit gene SCN1A in core severe myoclonic epilepsy in infancy (SMEI) and in borderline SMEI (SMEB). Epilepsia 2004; 45: 140-148.

42. Ohmori I, Ouchida M, Ohtsuka Y, Oka E, Shimizu K. Significant correlation of the SCN1A mutations and severe myoclonic epilepsy in infancy. Biochem Biophys Res Commun 2002; 295: 17-23.

43. Sun H, Zhang Y, Liu X, et al. Analysis of SCN1A mutation and parental origin in patients with Dravet syndrome. J Hum Genet 2010; 55: 421-427.

44. Xu X, Yang X, Wu Q, et al. Amplicon resequencing identified parental mosaicism for approximately $10 \%$ of "de novo" SCN1A mutations in children with Dravet syndrome. Hum Mutat 2015; 36: 861-872.

45. Harkin LA, McMahon JM, Iona X, et al. The spectrum of SCN1A-related infantile epileptic encephalopathies. Brain 2007; 130: 843-852.

46. Dlugos D, Ferraro T, Buono R. Novel de novo mutation of a conserved SCN1A amino-acid residue (R1596). Pediatr Neurol 2007; 37: 303-305.

47. Martin MS, Tang B, Papale LA, Yu FH, Catterall WA, Escayg A. The voltage-gated sodium channel Scn8a is a genetic modifier of severe myoclonic epilepsy of infancy. Hum Mol Genet 2007; 16: 2892-2899.

48. Ohmori I, Ouchida M, Miki T, et al. A CACNB4 mutation shows that altered $\mathrm{Ca}(\mathrm{v}) 2.1$ function may be a genetic modifier of severe myoclonic epilepsy in infancy. Neurobiol Dis 2008; 32: 349-354.

49. Singh NA, Pappas C, Dahle EJ, et al. A role of SCN9A in human epilepsies, as a cause of febrile seizures and as a potential modifier of Dravet syndrome. PLoS Genet 2009; 5: e1000649. 
50. Campbell IM, Stewart JR, James RA, et al. Parent of origin, mosaicism, and recurrence risk: probabilistic modeling explains the broken symmetry of transmission genetics. Am J Hum Genet 2014; 95: 345-359.

51. Goldmann JM, Wong WS, Pinelli M, et al. Parent-oforigin-specific signatures of de novo mutations. Nat Genet 2016; 48: 935-939.
52. Francioli L, Polak PP, Koren A, et al. Genome-wide patterns and properties of de novo mutations in humans. Nat Genet 2015; 47: 822-826.

53. Selmer K, Eriksson AS, Brandal K, Egeland T, Tallaksen C, Undlien DE. Parental SCN1A mutation mosaicism in familial Dravet syndrome. Clin Genet 2009; 76: 398-403. 